This document is the accepted manuscript version of the following article:

Dachraoui, W., Bodnarchuk, M. I., Voge1, A., Kovalenko, M. V., \& Erni, R. (2021). Unraveling the shell growth pathways of Pd-Pt core-she11 nanocubes at atomic level by in situ liquid cel1 electron microscopy. Applied Physics Reviews, 8(4), 041407 (8 pp.).

https://doi .org/10.1063/5.0059186

\title{
Unravelling the Shell Growth Pathways of Pd-Pt Core-Shell Nanocubes at Atomic Level by In Situ Liquid Cell Electron Micros- copy
}

\author{
Walid Dachraoui, ${ }^{1 *}$ Maryna I. Bodnarchuk ${ }^{2}$, Alexander Vogel ${ }^{1,3}$, Maksym V. Kovalenko $^{2,4}$, Rolf Erni $^{1 *}$ \\ ${ }^{1}$ Electron Microscopy Center, Empa-Swiss Federal Laboratories for Materials Science and Technology, Über- \\ landstrasse 129, CH-8600, Dübendorf, Switzerland. \\ ${ }^{2}$ Laboratory for Thin Films and Photovoltaics, Empa-Swiss Federal Laboratories for Materials Science and \\ Technology, Überlandstrasse 129, CH-8600, Dübendorf, Switzerland. \\ ${ }^{3}$ Department of Materials, ETHZ - Swiss Federal Institute of Technology Zürich, Leopold-Ruzicka-Weg 4, CH- \\ 8093, Zürich, Switzerland. \\ ${ }^{4}$ Laboratory of Inorganic Chemistry, Department of Chemistry and Applied Biosciences, ETH Zürich, CH-8093 \\ Zürich, Switzerland. \\ *Correspondence to: \\ E-mail: walid.dachraoui@empa.ch
}

\begin{abstract}
Understanding the formation of core-shell nanomaterials is decisive for controlling their growth, structure and morphology, which is particularly important in catalysis. As a promising material for photo catalysis application, Pd-Pt core-shell nanoparticles (NPs) have been in the spotlight for many years owing to their catalytic performance typically superior to that of pure Pt nanoparticles. The generation of ultra-thin Pt skins of only a few atomic layers on Pd nanoparticles has turned out to be extremely difficult because Pt tends to form islands during deposition instead of a continuous shell. Therefore, understanding the atomic mechanisms of shell formation is critical for atomic-scale design and control of the platinum shell. Here, by using in situ graphene-based liquid cell scanning transmission electron microscopy (STEM), the growth mechanisms of the Pt shell on Pd nanocubes (NCs) are studied in aqueous solution at the atomic level. Pd-Pt coreshell NPs are formed via two distinct mechanisms: (i) at low concentration of Pt atoms, an ultra-thin skin of only a few atomic layers is formed via atom-by-atom deposition and (ii) at higher concentration of Pt atoms, inhomogeneous islands and thick shells are formed via attachment of Pt clusters. Our study provides a route to control core-shell growth and helps us to understand the exact atomic mechanisms of Pt shell growth on Pd seeds.
\end{abstract}

Keywords. Platinum, Palldium, core-shell, Atomic mechanisms, liquid-phase, STEM. 


\section{INTRODUCTION}

Core-shell nanoparticles (NPs), consisting of two different metals, have attracted enormous attention in recent years because of their various technological applications. This interest is triggered by their distinctive physical and chemical properties, such as enhanced catalytic performance stability, improved thermal stability, and optical properties ${ }^{1-7}$. Synthesizing core-shell nanoparticles with well-controlled shell thickness and composition is of outmost importance in optimizing their reactivity ${ }^{8}$. Platinum is considered as a highly effective catalyst for oxygen reaction redaction (ORR) in proton-exchange membrane fuel cells (PEMFCs) ${ }^{9-11}$. However, its abundance in Earth's crust is extremely low, making its price double every decade because of the ever-growing demand. In order to increase the utilization efficiency while reducing the Pt content in catalysts, numerous methods have been explored to improve the specific and mass activity. Among them, the most promising approach is to deposit Pt atoms as ultrathin skins of only a few atomic layers on nanoparticles made of more abundant and/or less expensive metals, to form Pt-M core-shell structures ( $\mathrm{M}=\mathrm{Pd}, \mathrm{Fe}, \mathrm{Co}, \mathrm{Ni}$, etc) ${ }^{12-14}$. Not only can the core-shell Pt-M catalyst reduce the cost, but it also shows a greatly enhanced ORR activity, up to 22 times, relative to the pure Pt catalyst ${ }^{15}$. Due to their similarity in terms of lattice constant and chemical reactivity, Pd and Pt can be readily prepared as bimetallic core-shell nanocrystals with a singlecrystal structure and good stability ${ }^{16}$. Both theoretical and experimental studies have shown that combinations of Pd and Pt can be beneficial in terms of catalytic properties. Moreover, the ability of Pd to prevent Pt from corrosion by either sacrificing Pd or upshifting the dissolution potential of Pt, enhances the durability and stability of Pd-Pt core-shell nanoparticles acting as electro catalyst in various kinds of fuel cells ${ }^{17-27}$. Unfortunately, despite the great progress that has been made in the synthesis of Pd-Pt core-shell nanocrystals, deposition of Pt on Pd nanocrystals with precise control over the number atomic layers and the structure of the shell has been achieved with limited success. Controlled formation of ultra-thin skins of Pt, consisting of few atomic layers, have turned out to be extremely difficult, as Pt tends to form islands during deposition instead. For example, traditional atomic layer deposition (ALD) in gas phase and most of the solution-phase methods often generate Pt overlayers with polycrystalline structure and uneven thickness ${ }^{28}$. All hypotheses link the Pt islands formation to the conditions used for preparation, which are expected to be too mild to provide the thermochemical energy needed to break the strong Pt-Pt bonds. Moreover, the capping agents adsorbed on the nanoscale substrates tend to prevent Pt atoms from spreading across the entire surface of the substrate. The significant role played by chemical additives still remains to be a considerable challenge in order to precisely control the uniformity and the thickness of the Pt shell at the atomic scale. However, current research on Pd-Pt core-shell nanoparticles is mostly based on postreaction investigations ${ }^{29}$, which fail to unravel intermediate states. Therefore, the whole process of Pd-Pt core-shell formation is still unclear. For example, the study made by Xia and co-workers, where they were able to control the thickness of the Pt shell down to the atomic scale by adjusting the injection rate of the Pt precursor, revealed that the variation of the amount of Pt precursor enables the control of the thickness of the Pt shell from one to six atomic layers ${ }^{14}$. However, the mechanism of this deposition process was not well developed in addition to the lack of information about the exact mechanisms of island-type Pt shell formation. In order to better understand the mechanisms of the Pt shell formation on Pd and in order to precisely control the synthesis of these core-shell NPs, 
a method of continuous observation at the atomic level should be used to directly understand the complete formation process at the atomic scale of such complex nanostructures.

Recent developments in transmission electron microscopy and microfabrication provide new opportunities for imaging particles in a liquid environment ${ }^{30-44}$. However, silicon nitride-based liquid cells limit the attainable spatial resolution due to the thickness of the silicon nitride membranes (around $50 \mathrm{~nm}$ ) added to the thickness of the encapsulated liquid phase ranging from about $50 \mathrm{~nm}$ to $5 \mu \mathrm{m}^{45}$. Alternatively, non-commercial graphene liquid cells (GLCs) provide the opportunity to observe nanoparticles at the true atomic-level. Graphene sheets are composed of a few graphitic monolayers with an overall thickness below $1 \mathrm{~nm}$, allowing for imaging liquid samples and particles therein at the Ångström level ${ }^{46}$. The impermeability and the mechanical flexibility of graphene enables the entrapment of liquid nanoreactors without risk of leakage to the surrounding vacuum environment of the electron microscope. Moreover, the chemical neutrality and electrical conductivity of graphene enable the study of chemical reactions inside GLCs while mitigating radiolysis and charging effects ${ }^{47}$.

Here, we use graphene liquid cells to directly observe the formation process of Pd-Pt core-shell NPs at the atomic scale and to unravel two different shell growth modes. (i) In the first mode, Pt is directly deposited onto Pd NCs via atomic layer-by-layer deposition, or more precisely atom-by-atom deposition, where eventually a homogeneous thin shell is formed. (ii) In the second mode, Pt nanoparticles attach to Pd NCs via different processes such as oriented attachment and imperfect attachment, leading to inhomogeneous shells. The lattice strain for both types of core-shell structures is studied using geometric phase analysis (GPA).

\section{RESULT}

For seed materials, we synthesized cubic Pd nanoparticles (details in supporting information). Figure 1a shows typical aberration-corrected annular dark-field scanning TEM (ADF-STEM) images of the as synthesized Pd NPs materials. The Pd NPs represent an average particle size of $10 \mathrm{~nm}$, whereby $~ 90 \%$ are of cubic shape (denoted as cubic Pd NCs) while occasionally they were slightly elongated along one of the directions. This justifies that they are collectively called "nanocubes" in our discussion. We used energy-dispersive Xray spectroscopy (EDX) mapping of Pd elements to confirm that the formed nanocubes are composed of Pd. Atomic resolution ADF-STEM of one of the Pd nanocubes shown in the zoom-in of Figure $1 \mathrm{~b}$ indicates that the formed nanocubes are expectedly single crystals. The fast Fourier transform (FFT) pattern reveals that the inspected nanocube is a monocrystal oriented along the [100] direction corresponding to the face-centered-cubic (fcc) phase of bulk metallic Pd, as shown in the inset of Figure 1b. The majority of the nanocubes are oriented along their [100] zone axis because of their $\{100\}$ surface facets.

We used GLC-STEM to monitor the growth of Pt on top of Pd NCs while forming a core-shell structure. Dispersed Pd nanocubes in Pt precursor solution were encapsulated as tiny droplets between two sheets of graphene that were supported by holey carbon films on copper TEM grids (Figure 1c). Two concentrations of the Pt precursor $\left(\mathrm{Na}_{2} \mathrm{PtCl}_{4} \cdot 2 \mathrm{H}_{2} \mathrm{O}\right.$ in deionised water) were used, namely $1 \mathrm{mM}$ and $5 \mathrm{mM}$. Once the electron beam reduces the Pt precursor, metallic Pt is deposited onto the Pd NCs to eventually form a shell. 
Figures 1d and 1e show atomic-resolution ADF-STEM images of two typical Pd nanocubes coated with a Pt shell, corresponding to $1 \mathrm{mM}$ and $5 \mathrm{mM}$ Pt solution, respectively. The difference in atomic number (Pd: 46 and Pt: 78) allows the core and the shell to be distinguished clearly, despite the fact that the cell and the liquid phase reduce the chemical contrast. In both Pd-Pt core-shell examples shown in Figures $1 \mathrm{~d}$ and 1e, the Pt shell appears as relatively brighter region and the Pd core corresponds to the darker region, because the intensity is approximately proportional to $\mathrm{Z}^{1.7}$ (where $\mathrm{Z}$ is the atomic number) ${ }^{48}$. The ADF-STEM images confirm that the cubic shape was well preserved during the in-situ Pt deposition. Our atomic-resolution imaging further reveals that two types of Pt shells could be formed. Using the $1 \mathrm{mM}$ Pt solution, quite a thin but homogenous shell is formed (Figure 1d) while when using the $5 \mathrm{mM}$ Pt solution a rather thick shell, composed of inhomogeneous islands on top of the Pd nanocubes (Figure 1e), formed. To highlight the difference between the first and the second type of shell, schematic representations of both types are shown in Figures 1e and 1d. Moreover, Figures 1d and 1e (right) depict line profiles of EDX data recorded along the red line marked in the zoom in area of Figures $1 \mathrm{~d}$ and 1e, further confirming the elemental compositions and thickness for Pt shells and Pd core as inferred from the Z-contrast micrographs. These analyses indicate that there must be different mechanisms of Pd-Pt core-shell NCs formation at the atomic level, which gives rise to two different Pt shells, either a thin homogeneous skin of atomic layers of Pt or an inhomogeneous, rather thick shell consisting of different islands. In order to explore these mechanisms, we tracked the shell formation at the atomic scale in real time using high-resolution ADF-STEM imaging.
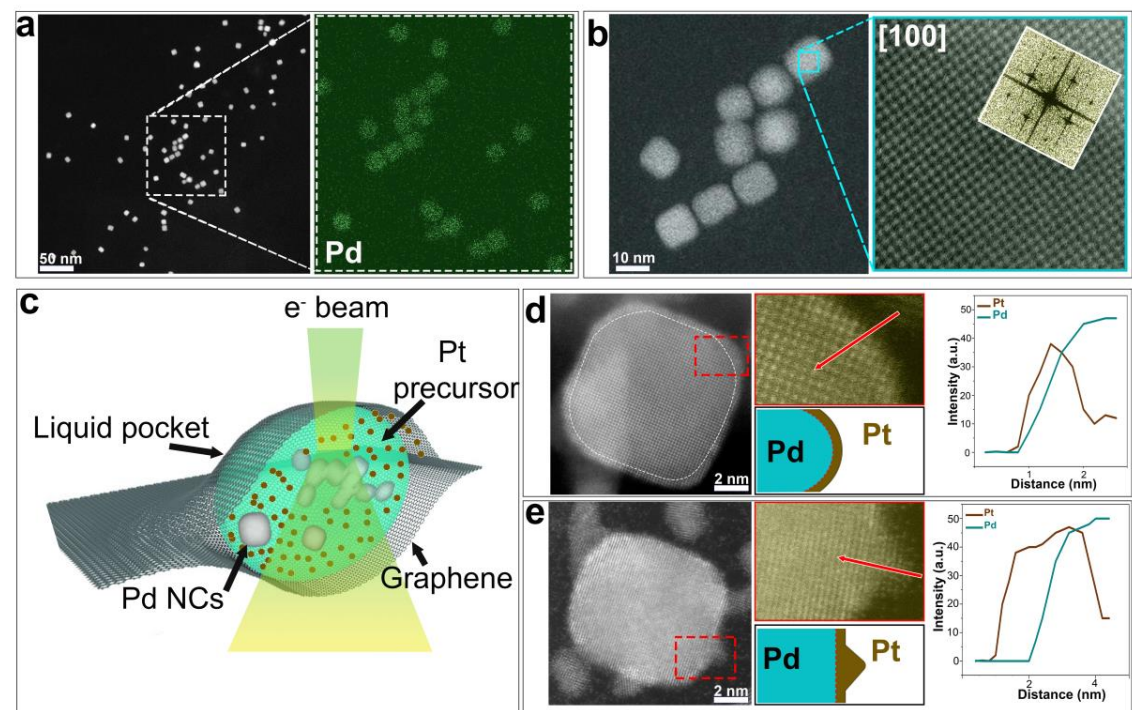

Figure 1. Pd NCs core and Pt shell STEM analysis (a) Overview ADF-STEM image of the synthesized Pd NCs with the corresponding EDX elemental map of Pd. (b) ADF-STEM image of the cubic Pd nanocrystals and atomicresolution ADF-STEM images of a single Pd NCs with its corresponding FFT pattern. (c) Schematic illustration of a GLC encapsulation of Pd NCs with a solution of Pt precursor illuminated with an electron beam. (d) Atomicresolution ADF-STEM image taken from a single Pd nanocube with thin layer of Pt shell formed in a solution of 
$1 \mathrm{mM}$. The zoom-in shows clearly the formed Pt shell confirmed by EDX line scan analysis along the red arrow. (e) Atomic-resolution ADF-STEM image taken from a single Pd nanocube with of Pt shell islands formed in a solution of $5 \mathrm{mM}$. The zoom-in shows clearly the formed Pt shell confirmed by EDX line scan analysis along the red arrow.

Atom-by-atom deposition. Here we encapsulate the Pd NCs with $1 \mathrm{mM}$ of Pt precursor. Since the liquid pocket is very thin (below $50 \mathrm{~nm}$ ) the created and reduced Pt atoms in the surrounding of the Pd nancubes are easily imaged (Figure S2 in supporting information) using an electron beam with an electron dose rate of about $2 \times 10^{4}$ electrons $/ \AA^{2}$ s. The growth of a Pt shell on top of the Pd NCs, resulting from the electron irradiation, was observed. Figure 2 a shows the time-lapse atomic-resolution ADF-STEM images of Pt shell overgrowth on a single Pd NC projected along the [100] direction (See movie S1). At $t=40 \mathrm{~s}$ a border of increased brightness around the Pd nanocube starts to be clearly visible, which corresponds to the Pt shell formed around the Pd NC. With increasing time, the brightness as well as the thickness of the shell increases. While after $10 \mathrm{~s} \mathrm{~T}_{1}=11.0 \mathrm{~nm}$ and $\mathrm{T}_{2}=11.2 \mathrm{~nm}$, these distances increase to become $\mathrm{T}_{1}=11.3 \mathrm{~nm}$ and $\mathrm{T}_{2}=$ $11.5 \mathrm{~nm}$ at $t=240 \mathrm{~s}\left(\mathrm{~T}_{1}\right.$ and $\mathrm{T}_{2}$ define the thickness of the Pd NC along the two directions as shown in Figure 2a). In order to further develop the mechanism of the shell growth, a zoom-in on the sides of the Pd NC was followed in real time. Figure $2 b$ shows the time-lapse of the region highlighted by the yellow box in Figure $2 \mathrm{a}$, where the atomic images corresponding to both types of elements ( $\mathrm{Pt}$ and $\mathrm{Pd})$ are clearly visible and the difference between them is distinguishable (Pt: brighter, Pd: darker). According to the atomic-resolution ADF-STEM images shown in Figure 2b (see Movie S1 for more details), we find that the Pt shell was formed by successive deposition of individual atoms at the $\operatorname{Pd}\{100\}$ surfaces. From the time lapse in Figure $2 \mathrm{~b}$ and the region highlighted by the white box, it can be seen that a new atomic layer with a relatively brighter contrast than the core material starts to form from $t=40 \mathrm{~s}$. The addition of Pt atoms from the surrounding liquid is clearly visible. After $200 \mathrm{~s}$, a complete projected Pt atomic layer is formed at the Pd-liquid interface and a second layer of Pt atoms starts on top of the previous one. In this step, the thin Pt shell tends to grow by attracting atoms from other Pt sources, such as isolated Pt atoms or from surrounding clusters and unstable NPs via Ostwald ripening. This possibility is supported by our in situ STEM images in Figure S3, which reveal the detachment of atoms from small Pt NPs in the liquid phase to eventually attach to the surface of the Pt-covered Pd NC. A schematic illustration showing the deposition of Pt atoms on Pd cubes is shown in Figure 2b. Additional examples of this shell growth mechanism, based on the deposition of individual Pt atoms, are provided in Figure S4a of the supporting information.

In order to confirm that the electron beam reduces the Pt precursor before forming the shell, we performed one reference experiment. A solution of Pt precursor without Pd cubes is encapsulated then exposed to the electron beam with the same electron dose. In this condition, we observed clearly visible Pt atoms which coalesce and form small Pt clusters (more details in Figure S5). 

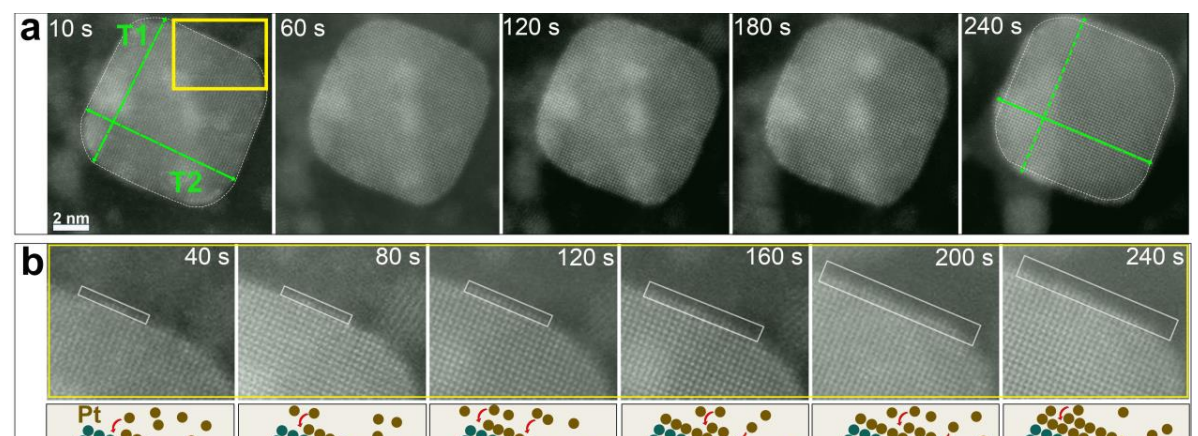

$120 \mathrm{~s}$

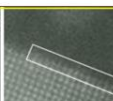

$160 \mathrm{~s}$

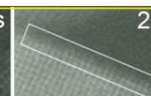

$200 \mathrm{~s}$

$240 \mathrm{~s}$

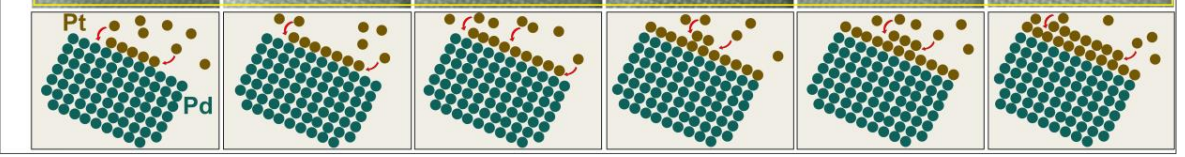

Figure 2. Growth of Pt shell on the Pd NC via Pt atom deposition. (a) Time-lapse series of ADF-STEM images shows the growth of the Pt shell on the Pd NC forming a thin skin of few atomic layers. (b) Top: Atomic mechanisms of Pt atoms deposition on one of the sides of Pd NC highlighted by the yellow box in (a), where successive atom-by-atom deposition is visible. Bottom: A schematic illustration showing the deposition of Pt atoms on Pd cubic seeds. Images are shown in false color.

Attachment of nanoparticles. By increasing the Pt concentration in the graphene encapsulated liquid phase to $5 \mathrm{mM}$ Pt precursor, we favored the growth of Pt nanocrystals prior to forming a shell around the Pd seed crystals. Figure 3a shows sequential atomic-resolution ADF-STEM images of the nucleation of a selected Pt nanoparticle in the surrounding area of a Pd NC. Time $t=0 \mathrm{~s}$ in the Movie S2 and in Figure 3a represents the starting point of imaging in that particular area, after having optimized the imaging conditions in a different area to minimize the beam effect in the area of observation. Irradiation with an electron dose rate of about $2 \times 10^{4}$ electrons $/ \AA^{2}$ s reduces the Pt precursor as in the previous case. Compared to the case with lower precursor concentration, small particles of increased brightness have formed in neighbouring areas and migrate via the liquid to the imaging area, while the bright dots in Figure 3a correspond to freshly reduced Pt atoms. After $10 \mathrm{~s}$, the Pt atoms start aggregating to form a stable cluster. Then, Pt atoms from the surrounding liquid rapidly attach to this nanocluster. We followed the evolution of three clusters highlighted with a red circle in the image at $t=10 \mathrm{~s}$ in Figure 3a. These three clusters continue increasing in size via atomic attachment until they start coalescence at $t=70 \mathrm{~s}$ to end up with a mature well crystallized NP of around $2 \mathrm{~nm}$ in size (also see Movie S2 for details). After formation of many such NPs with different sizes and shapes in the surrounding area of the Pd NC an attachment process starts, where the Pt NPs approach the Pd NC and start attaching to its $\{100\}$ faces and the corresponding corners and edges between them. Figure $3 b$ shows a typical atomic-resolution ADF-STEM micrograph of a Pd NC projected along the [100] direction along with small Pt NPs grown in its surrounding liquid area. We highlighted three zones with squares in white, yellow and red colors, near the Pd-liquid interface where Pt nanoparticles are present. On the right hand side of Figure $3 b$, the corresponding sequences of images documenting the trajectories of these Pt NPs are shown which reveal the details of the attachment process in real time (more details in Movie S3: white, Movie S4: yellow, and Movie S5: red). In these three examples, tiny Pt clusters of 1 to $2 \mathrm{~nm}$ in size start to coalesce to form 
bigger Pt nanocrystals. Thereafter these Pt nanocrystals mature and improve in crystallinity. In the next step, the Pt NPs approach and attach to the surface of the Pd NCs. Finally at $t=90 \mathrm{~s}$, an inhomogeneous Pt shell is formed that consists of islands of attached Pt nanoparticles. During the formation of the Pt shell mediated by Pt NPs, the predominant phenomena were the high-frequency coalescence and aggregation of Pt clusters and nanoparticles that led to an increase of the Pt NPs size while their number decreases as shown in Figure S6, which was triggered by Brownian-type or electron-beam induced particle motion. More examples of Pt NPs coalescence induced Pt shell growth are shown in Figure S4b in the supporting information.

In the next step, we aim at revealing the exact atomic mechanism of particle attachment that leads to the formation of the inhomogeneous Pt shell. Figure 3c shows two ADF-STEM image sequences extracted from Movies S3 and S5. The Pt crystal shown in the first sequence is the same crystal as highlighted by the white square in Figure $3 \mathrm{~b}$. At the beginning, four Pt nanoparticles coalesce to form a bigger, elongated Pt NP after random and high speed movement, see first row in Figure 3b at 70 and $100 \mathrm{~s}$ (more details in Figure S7). This crystal is then involved in an oriented attachment process with the Pd NC, see first row in Figure 3c. Initially the Pt NP gets in contact with one of the facets of the Pd NC, where a neck formed. Then the Pt NP experiences a large, apparently random and sudden rotation. When the Pt particle is projected along its [100] direction, it starts to rotate in clockwise direction, indicated by red arrows, until its $\{100\}$ facet is perfectly aligned with the $\{100\}$ surface of the Pd NC in Figure 3c. Once these crystal facets are properly aligned in respect to each other, the connective neck between the particles vanishes through a rapid diffusion of surface atoms, leading to the formation of brighter atomic layers corresponding to a thin Pt shell on the surface of the Pd nanocrystal, particularly in the surrounding area of the attached Pt island. This is observable by a shell of brighter contrast that appears and increases in thickness during the coalescence (designed by blue arrows in Figure 3c). The second sequence (second row in Figure 3c) is a representative example of an imperfect attachment, where a Pt nanoparticle is involved in two types of coalescence; it first attaches to the Pd NC and then coalesce with a second Pt nanoparticle of similar size. The coalescence of the Pt NPs induces defects such as twin boundaries as indicated in the second image of the sequence. Thereafter, the attachment with the Pd NC continues via a defect elimination process until the particle's $\{100\}$ facets are perfectly aligned with the $\{100\}$ of the Pd NC. Then the NP quickly adsorbs on the surface of the Pd NC (yellow arrows).

In order to confirm that the shell growth occurs only in the liquid phase and in presence of a Pt precursor, we performed two control experiments. Pd NCs were deposited on graphene layers in dry mode without Pt precursor then exposed to the electron beam with the same electron dose for $400 \mathrm{~s}$, and graphene encapsulated Pd NCs with its solvent solution was exposed to the beam for $400 \mathrm{~s}$. In both cases, no obvious changes and modifications occurred of the Pd NCs and of their surrounding areas (more details in Figure S8). 

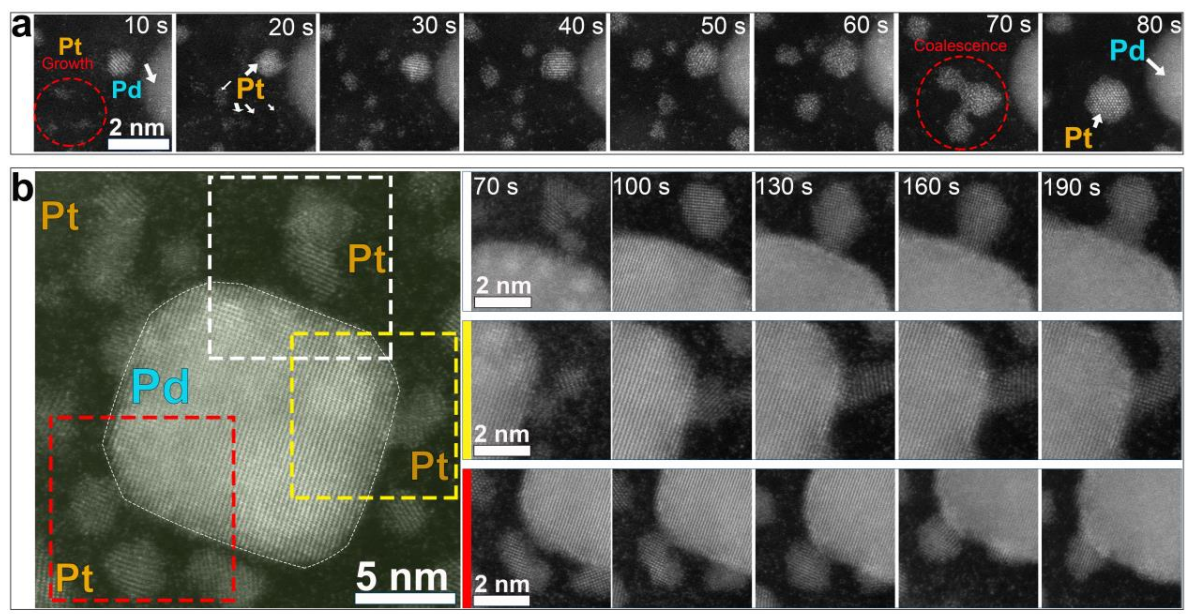

$190 \mathrm{~s}$
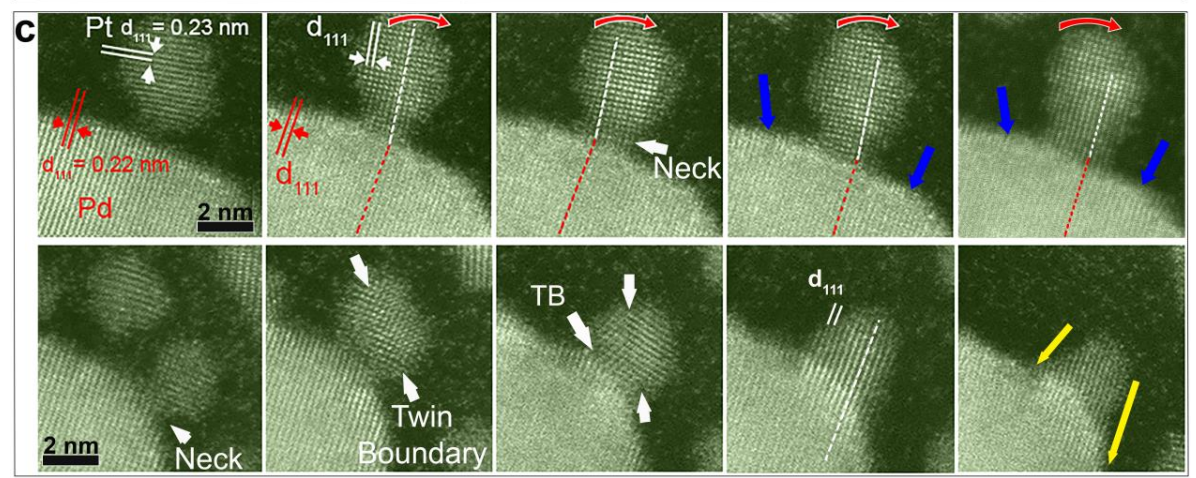

Figure 3. Growth of Pt NPs induced shell growth on the Pd NCs via Pt nanoparticle attachment. (a) Time-lapse series of ADF-STEM images shows the growth of the Pt nanoparticle in the surrounding area of a Pd NC. (b) Left: typical atomic resolution ADF-STEM image of a Pd NC surrounded by Pt nanoparticles. Right: three time-lapse series of ADF-STEM images showing the attachment of three Pt nanoparticles highlighted with squares in the left image. (c) Time-lapse series of atomic-resolution ADF-STEM images showing oriented attachment and imperfect particle attachment.

\section{DISCUSSION}

With increasing concentration of the Pt precursor inside our GLCs nonreactors with the same electron dose rate $\left(2 \times 10^{4}\right.$ electrons $\left./ \AA^{2} \mathrm{~s}\right)$, the formed Pt shell changes from a thin skin of Pt atomic layers to an inhomogeneous layer of Pt islands attached to the surface of the Pd NC. We observed that with $1 \mathrm{mM}$ the formed Pt atoms migrate through solution and attach to the Pd NC. This can be related to the low concentration of Pt atoms in the solution that does not allow nucleation of Pt nanoparticle in the solvent. Instead individual Pt atoms or ultra-small Pt clusters are formed that lead to the formation of a thin homogeneous Pt shell on the $\mathrm{Pd}$ NC. This is consistent with the study made by Xia and co-workers who showed that slowly injecting Pt 
precursor in the solution to keep the concentration of Pt atoms at low level prevents self-nucleation of Pt and results in Pt atoms that undergo a heterogeneous nucleation on the Pd seeds ${ }^{14}$. Our real time observations reveal that the atomic deposition indeed takes place via atom-by-atom deposition on the $\operatorname{Pd}\{100\}$ faces and the corners and edges between them. This mechanism leads to a homogeneously thin Pt shell. This is in contrast to the suggested deposition process in that $\mathrm{study}^{14}$, which was proposed to start at corners with subsequent diffusion to edges and faces. In our study, we did not find any indications for distinctly preferred sites where the shell growth nucleates. In addition, our study goes further and shows that increasing the concentration of Pt in the nanoreactor does not primarily lead to an increase in the thickness of the Pt shell, but instead leads to the nucleation of Pt nanoparticles in the solution that attach to the Pd NC, which confirms the previously discussed suggestion ${ }^{14}$. Indeed, the Pt nanoparticles involved in the attachment processes form an inhomogeneous Pt shell with Pt islands on the surface of Pd NCs. This result based on real-space atomicscale observation confirms that the concentration of Pt precursor plays a critical role for the final Pt shell on Pd-Pt structure. Both shell growth pathways are simultaneously observed. However, which of the two pathways dominates depends on the initial concentration of the Pt precursor. Thus, the variation in concentration of Pt precursor favors one process compared to the other depending on the initial concentration of Pt precursor. This is indeed a very general observation; there are often competing processes simultaneously active and it is a local variable that essentially defines which of the competing processes dominates the actual reaction and defines its pathway as well as the final product. In our case, the precursor concentration turns out to be the critical variable.

Due to the fact that the lattice parameter of bulk Pt is roughly $1 \%$ larger than the one of Pd, the deposition of Pt onto the Pd NC could lead to a lattice mismatch between core and shell region, which induces strain. This can affect the electronic properties of the Pt shell, which is important for its catalytic activity. We analyzed the lattice strain for both types of core-shell structures produced in our in situ LC-STEM study ${ }^{49}$. We use geometric phase analysis (GPA) based on atomic resolution ADF-STEM images of Pd-Pt core-shell nanocubes formed via Pt atomic deposition (figure 4a) and a core-shell nanocube formed via Pt NPs attachment (Figure 4b). GPA was performed on nanocubes with comparable size and the false colored images represent the in-plane $\left(\varepsilon_{x x}\right)$ strain field determined by GPA showing lattice deformation relative to a reference area, in our case the region marked by the red circles in the Pd regions (Figures 4a and 4b). Figure S9 shows the $\varepsilon_{y y}$ and $\varepsilon_{x y}$ strain field determined by GPA. The red coloring on the particle represents an expansive lattice deformation relative to the reference area and the blue coloring represents a contraction, respectively. The oscillation in coloring observable in the GPA maps may originate from an amorphous shell that impacts the clarity of the atomic resolution images and from the chosen masks applied to select spots in the FFT pattern 50,51. Moreover, where there is no crystalline lattice present, as in the surrounding of the NP, the GPA analysis does not provide any interpretable information. If the Pt-shell perfectly adopts the spacing of the Pd seed crystal via epitaxial relaxation, then there should be no obvious regions of red or blue on the shell region surrounding the Pd NC, indicating minimal or no lattice deformation relative to the Pd core. However, if the Pt shell does not adopt the spacing of the Pd NC, then in the GPA maps there should be regions of red or blue color on the shell region surrounding the $\mathrm{Pd} \mathrm{NC}{ }^{47}$.

The $\varepsilon_{\mathrm{xx}}$ strain field for a representative Pd-Pd nanocube formed in $2 \mathrm{mM}$ of Pt precursor with a shell consisting of a few atomic layers of Pt is shown in Figure 4a and reveals minimal red coloration on the Pt-shell region, 
indicating that there is minimal deformation of the Pt shell relative to the Pd core. The Pt shell adopts the lattice of the Pd NC and is thus strained. However, for the Pd-Pt core-shell structure grown via Pt NPs attachment in $5 \mathrm{mM}$ of Pt precursor in Figure 4b, the GPA analysis reveals an expansion of the lattice constant in the Pt shell, indicated by the red-colored areas in the Pt shell of Figure 4b. In this case, the Pt shell thus does not adopt, or at least not fully adopt, the lattice constant of the Pd seed crystal.
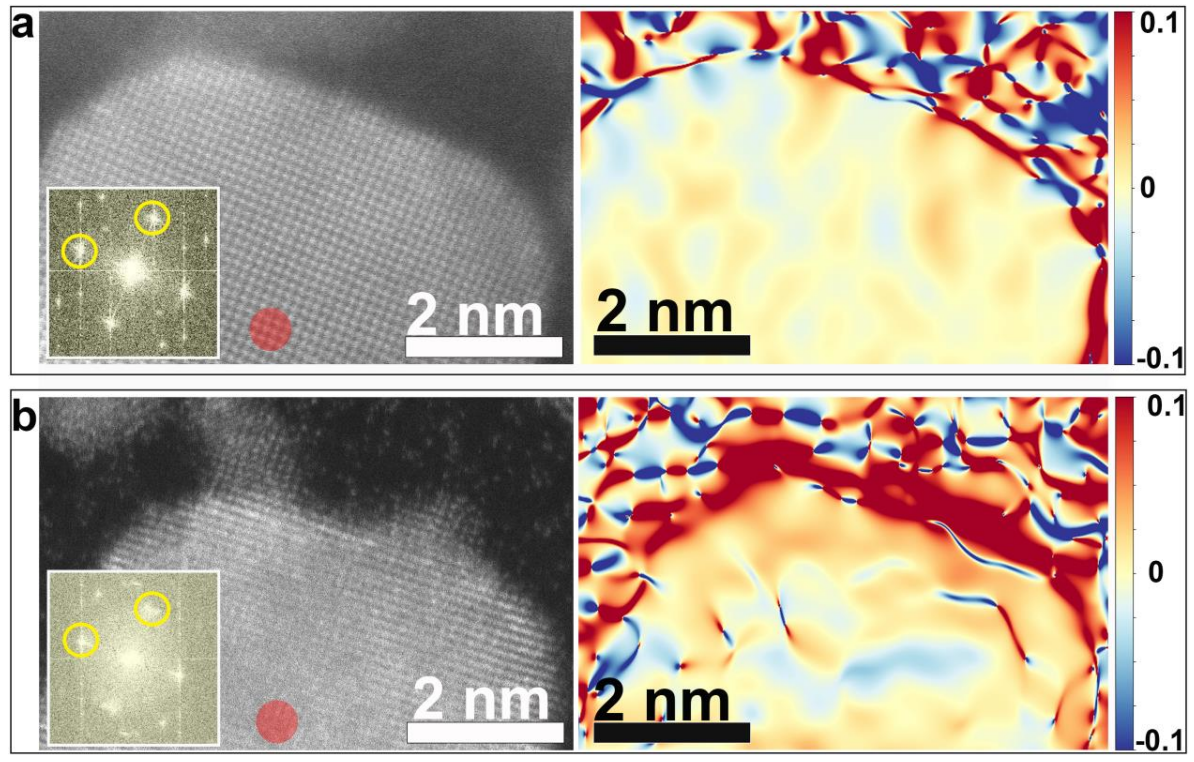

Figure 4. Atomic-resolution ADF-STEM images and corresponding GPA analysis for (a) Pd-Pt core-shell nanocube with few atomic layers of Pt and (b) Pd-Pt core-shell nanocube with inhomogeneous Pt island shell. The first column consists of the ADF-STEM images and its FFT patterns. The second column consist of the corresponding GPA color maps which correspond to the in-plane strain $\left(\varepsilon_{x x}\right)$ field (which is the strain in the plane of the crystal face) where the intensity scale shows relative deformation.

\section{SUMMARY}

According to our in situ observations at atomic scale in aqueous solution, the formation of the thin shell consisting of Pt atomic layers and thick inhomogeneous Pt shells with distinct islands are formed via two different pathways and atomic mechanisms, as illustrated in Figure 5. First, at low Pt concentration in the precursor solutions, the Pt shell grows by attracting individual Pt atoms or clusters in solution. At higher Pt concentration in the precursor solution, Pt nanoparticles nucleate and grow in the solvent and attach to the Pd NC, eventually leading to an inhomogeneous shell. Both growth pathways can produce Pd-Pt core-shell nanoparticles. However, the lattice relaxation of both of them are different, which can affect their properties. 


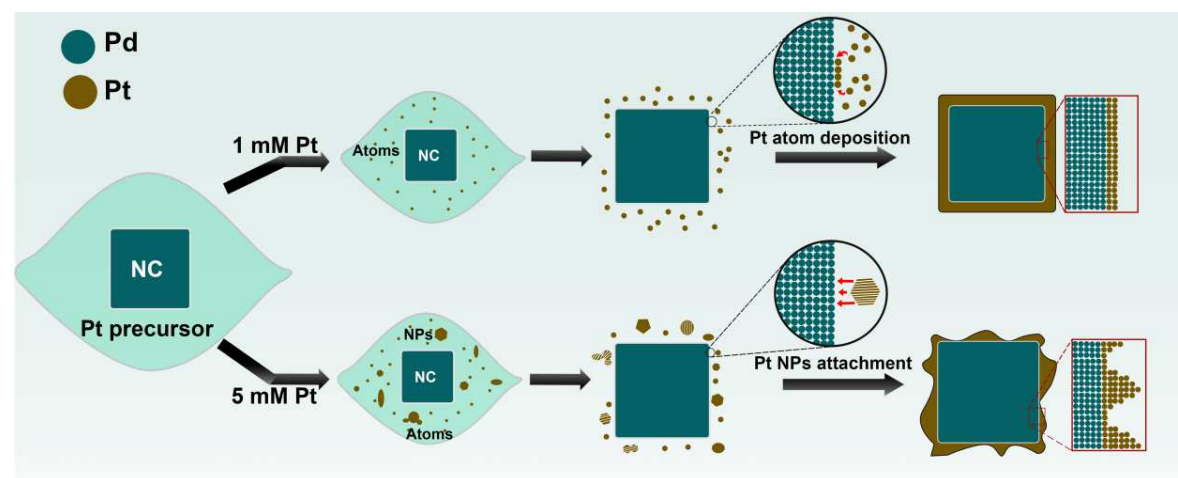

Figure 5. Schematic illustration of the formation pathways of Pt shells on Pd NCs: (i) Low Pt precursor concentration: atom deposition induced formation of a thin atomic layer Pt shell and (ii) high Pt precursor concentration: Pt nanoparticles nucleate in the solvent and lead to a thick inhomogeneous shell of Pt islands.

In conclusion, we used GLCs enabling imaging at the atomic scale in liquid mode to explore the atomic mechanisms of the growth of Pt shells on Pd NCs in detail. Our in situ observations revealed that the Pt solvent concentration plays a crucial role in controlling the growth mode of the Pt shell. We unravel two growth pathways of the Pt shell on Pd seed crystals. By keeping the concentration of $\mathrm{Pt}$ atoms in the reaction solution at low level, we prevent the nucleation of Pt nanoparticles in the solvent, and then the Pt atoms attach one by one to the Pd NC forming ultrathin, homogeneous shells consisting of few atomic layers of Pt. At increased concentration of $\mathrm{Pt}$ in the solvent, $\mathrm{Pt}$ particles nucleate in the solvent and form stable nanoparticles in the precursor solution, which attach to the Pd NC to form an inhomogeneous Pt shells. The attachment of Pt NPs is found to occur via different processes such as oriented attachment and imperfect attachment. Moreover, while the homogeneous Pt shell adopts the lattice parameter of the Pd seed crystal and is thus strained, the Pt islands of the inhomogeneous Pt shell show a larger lattice parameter than the seed crystal and are thus in a state closer to bulk Pt. These in situ experiments allow us to investigate the exact atomic mechanisms of Pd-Pt core-shell formation. Our findings open the way for better control in the synthesis and application of Pt-based core-shell nanomaterials.

\section{ASSOCIATED CONTENT}

Supporting Information 
Details for the sample preparation, GLCs fabrication and methods for analyses, Figures S1-S8 (PDF).

In-situ ADF-STEM observation of Pd NC in $1 \mathrm{mM}$ Pt solution undergoing the atom-by-atom deposition of Pt atoms(AVI), In-situ ADF-STEM observation of Pt nanoparticle growth in $5 \mathrm{mM}$ Pt solution in the surrounding area of Pd NCs (AVI), In-situ ADF-STEM observation of Pt NP involved in coalescence with Pd NC in $5 \mathrm{mM} \mathrm{Pt}$ solution to form inhomogeneous shell (AVI) In-situ ADF-STEM observation of Pt NP involved in coalescence with Pd NC in 5m M Pt solution to form inhomogeneous shell (AVI), In-situ ADF-STEM observation of Pt NP involved in coalescence with Pd NC in $5 \mathrm{mM}$ Pt solution to form inhomogeneous shell (AVI).

\section{AUTHOR INFORMATION}

\section{Corresponding Author}

*Email: walid.dachaoui@empa.ch

*Email: rolf.erni@empa.ch

\section{ORCID}

Walid Dachraoui: 0000-0001-7599-5856

Rolf Erni: 0000-0003-2391-5943

\section{Authors' Contributions}

W.D. and R.E. conceived the idea and wrote the manuscript. W.D. fabricated the liquid cells, performed the liquidcell experiments and realised the in-situ STEM characterizations. W.D. and A.V carried out the data analysis. M.B and M.K. synthesised nanomaterials. R.E supervised the project. All authors discussed the results and commented on the manuscript.

Notes

The authors declare no competing interests.

\section{ACKNOWLEDGMENTS}

This work received funding from European Research Council (ERC) under the EU's Horizon 2020 research and innovation program under grand No. 681312. M.B acknowledge the funding received from Swiss National Science Foundation (grant number 200021_192308, project Q-Light).

\section{DATA AVAILABILITY}

The data that support the findings of this study are available from the corresponding author upon reasonable request

\section{REFERENCES}

${ }^{1}$ L. Bu, N. Zhang, S, Guo, X. Zhang, J. Li, J. Yao, T. Wu, G. Lu, JY. Ma, D. Su, X. Huang, Science. 6318, 1410 (2016).

${ }^{2}$ R. S. Ghosh, T. T. Le, T. Terlier, Ji. D. Rimer, M. P. Harold, D. Wang, ACS Catal. 10, 3604 (2020).

${ }^{3}$ W. Luc, C. Collins, S. Wang, H. Xin, K. He, Y. Kang, F. Jiao, J. Am. Chem. Soc. 139, 1885 (2017).

${ }^{4}$ Gilroy. K. D.; Ruditskiy. A.; Peng. H. C., D. Qin Y. Xia. Chem. Rev. 116, 10414 (2016).

${ }^{5}$ X. Zhu, X. Zhuo, Q. Li, Z. Yang, J. Wang, Adv. Funct. Mater. 26, 341(2016).

${ }^{6}$ M. Mayer, L. Scarabelli, K. March, T. Altantzis, M. Tebbe, M. Kociak, S. Bals, F. J. G. D. Abajo, A. Fery, L. M. Liz-Marzán, Nano Lett. 15, 5427 (2015).

${ }^{7}$ C. Chen, Y. Kang, Z. Huo, Z. Zhu, W. Huang, H. Xin, J. D. Snyder, D. Li, J. A. Herron, M. Mavrikakis, M. Chi, K. L. More, Y. Li, N. M. Markovic, G. A. Somorjai, P. Yang, V. R. Stamenkovic, Science. 343, 1339 (2014).

${ }^{8}$ L. Kuai, S. Wang, B. Geng, Chem Commun. 47, 6093 (2011).

${ }^{9}$ B. C. H. Steele, A. Heinzel, Nature. 414, 345 (2001).

${ }^{10}$ S. Guo, S. Zhang, S. Sun, Angew. Chem., Int. Ed. 52, 8526 (2013).

${ }^{11}$ M. K. Debe, Nature. 486, 43 (2012).

${ }^{12}$ Y. Liu, D. Gokcen, U. Bertocci, T. P. Moffat. Science. 338, 1327 (2012).

${ }^{13}$ C. Gao, Z. Lu, Y. Liu, Q. Zhang, M. Chi, Q. Cheng, Y. Yin, Angew. Chem. Int. Ed. 51, 5629 (2012). 
${ }^{14}$ S. Xie, S.-I. Choi, N. Lu, L. T. Roling, J. A. Herron, L. Zhang, J. Park, J Wang, M. J. Kim, Z. Xie, M. Mavrikakis, Nano Lett. 14, 3570 (2014).

${ }^{15}$ H. A. Gasteiger, S. S, Kocha, B. Sompalli, F. T. Wagner, Appl. Catal. B. 56, 9 (2005).

${ }^{16}$ H. Zhang, M. Jin, Y. Xia, Chem. Soc. Rev. 41, 8035 (2012).

${ }^{17}$ Y. Liu, D. Gokcen, U. Bertocci, T. P. Moffat, Science. 338, 1327 (2012).

${ }^{18}$ C. Gao, Z. Lu, Y. Liu, Q. Zhang, M. Chi, Q. Cheng, Y. Yin, Angew. Chem. Int. Ed. 51, 5629 (2012).

${ }^{19}$ E. Toyoda, R. Jinnouchi, T. Ohsuna, T. Hatanaka, T. Aizawa, S. Otani, Y. Kido, Y. Morimoto, Angew. Chem. Int. Ed. 52, 4137 (2013).

${ }^{20}$ R. R. Adzic, J. Zhang, K. Sasaki, M. B. Vukmirovic, M. Shao, J. X. Wang, A. U. Nilekar, M. Mavrikakis, J. A. Valerio, F. Uribe, Top. Catal. 46, 249 (2007).

${ }^{21}$ V. R. Stamenkovic, B. Fowler, B. S. Mun, G. Wang, P.N. Ross, C. A. Lucas, N. M. Markovic, Science. 315, 493 (2007).

${ }^{22}$ P. Strasser, S. Koh, T. Anniyev, J. Greeley, K. More, C. Yu, Z. Liu, S. Kaya, D. Nordlund, H. Ogasawara, M. F. Toney, A. Nilsson, Nat. Chem. 2, 454 (2010).

${ }^{23}$ J. R. Kitchin, J. K. Nørskov, M. A. Barteau, J. G. Chen, Phys. Rev. Lett. 93, 156801 (2004).

${ }^{24}$ B. Lim, M. Jiang, P. H. C. Camargo, E. C. Cho, J. Tao, X. Lu, Y. Zhu, Y. Xia, Science. 324, 1302 (2009).

${ }^{25}$ J. X. Wang, H. Inada, L. Wu, Y. Zhu, Y. Choi, P. Liu, W.-P. Zhou, R. R.J. Adzic, Am. Chem. Soc. 131, 17298 (2009).

${ }^{26}$ X. Wang, Y. Orikasa, Y. Takesue, H. Inoue, M. Nakamura, T. Minato, N. Hoshi, Y. Uchimoto, J. Am. Chem. Soc. 135, 5938 (2013).

${ }^{27}$ J. Greeley, J. K. Nørskov, Electrochem. Acta. 52, 5829 (2007).

${ }^{28}$ C. C. Yang, Z. T. Liu, Y. P. Lyu, C. L. Lee, Shell-Thickness-Controlled Synthesis of Core-Shell Pd@Pt Nanocubes and Tuning of Their Oxygen Reduction Activities. J. Electrochem. Soc. 164, 112 (2017).

${ }^{29}$ X. Wang, SI. Choi, LT. Roling, M. Luo, C. Ma, L. Zhang, M. Chi, J. Liu, Z. Xie, JA. Herron, M. Mavrikakis, Y. Xia, Nat Commun. 6, 7594 (2015).

${ }^{30}$ A. De Clercq, W. Dachraoui, O. Margeat, K. Pelzer, C. R. Henry, S. Giorgio, J. Phys. Chem. Lett. 5, 2126 (2015).

${ }^{31}$ B. Song, K. He, Y. Yuan, S. Sharifi-Asl, M. Cheng, J. Lu, W. A. Saidi, R. Shahbazian-Yassar, Nanoscale. 10, 15809 (2018).

${ }^{32}$ L. Junjie, C. Jiangchun, H. Wang, C. Na, Z. Wang, L. Guo, F. L. Deepak, Adv. Sci. 5, 1700992 (2018).

${ }^{33}$ M. Wand, C. Park, T. J. Woehl, Chem. Mater. 30, 7727 (2018).

${ }^{34}$ M. H. Nielsen, D. Li, H. Zhang, S. Aloni, T. Y. J. Han, C. Frandsen, J. Seto, J. F. Banfield, H. Cölfen, J. J. De Yoreo, Microsc. Microanal. 20, 425 (2014).

${ }^{35}$ W. Wie, H. Zhang, W. Wang, M. Dong, M. Nie, L. Sun, F. Xu, ACS Appl. Mater. Interfaces. 11, 24478 (2019).

${ }^{36}$ T. Y. Ahn, S. P. Hong, S. Kim, Y. W. Kim, RSC Adv. 5, 2342 (2015).

${ }^{37}$ J. Y. Cheong, J. H. Chang, S. J. Kim, C. Kim, H. K. Seo, J. W. Shin, J. M. Yuk, J. Y. Lee, D. Kim, Microsc. Microanal. 23, 1107 (2017).

${ }^{38}$ E. Javon, M. Gaceur, W. Dachraoui, O. Margeat, J. Ackermann, M. I. Saba,.; P. Delugas, A. Mattoni, S. Bals, G. Van Tendeloo, ACS Nano. 5, 3685 (2015).

${ }^{39}$ E. Sutter, P. Sutter, A. V. Tkachenko, R. Krahne, J. de Graaf, M. Arciniegas, L. Manna, Nat Com. 7, 11213 (2016).

${ }^{40}$ M. A.Touve, A. S. Carlini, N. C. Giannesch, Nat Comm. 10, 4837 (2019).

${ }^{41}$ H. Wang, B. Li, Y. J. Kim, O. H. Kwon, S. Granick, App Phy Sci. 117, 1283 (2020),

${ }^{42}$ D. Elgrabli, W. Dachraoui, C. Ménard-Moyon, X. J. Liu, D. Bégin, S. Bégin-Colin, A. Bianco, F. Gazeau, D. Alloyeau, ACS Nano. 10, 10113 (2015).

${ }^{42}$ G. Z. Zhu, S Y. J. Prabhudev, C. M. Gabardo, G. A. Botton, L. Soleymani, J. Phys. Chem. C. 118, 22111 (2018).

${ }^{43}$ V. Beermann, M. E. Holtz, E. Padgett, J. F. de Araujo, D. A. Muller, P. Strasser, Energy Environ. Sci. 12, 2476 (2019).

${ }^{44}$ M. R. Hauwiller, J. C. Ondry, A. P. Alivisatos, Journal of Visualized Experiments. 135, 2 (2018).

${ }^{45}$ S. Pu, C. Gong, A. Robertson, R. Soc. Open Sci. 7, 191204 (2020).

${ }^{46}$ W. Dachraoui, D. Keller, TR. Henninen, OJ. Ashton, R. Erni, Nano Lett. 21, 2861(2021).

${ }^{47}$ C. Wang, T. Shokuhfar, R.F. Klie, Adv. Mater. 28, 7716 (2016).

${ }^{48}$ P.D. Nellist, S.J. Pennycook, Advances in Imaging and Electron Physics. 113, 147 (2000).

${ }^{49}$ J. T. L. Gamler, A. Leonardi, X. Sang, K. M. Koczkur, R. R. Unocic, M. Enge, S. E. Skrabalak, Nanoscale Adv. 2, 1105 (2020).

${ }^{50}$ J. Chung, L. Rabenberg, Ultramicroscopy. 108, 1595 (2008).

${ }^{51}$ M.J. Hÿtch, E. Snoeck, R. Kilaas, Ultramicroscopy. 74, 131 (1998). 